\title{
Characteristics of daily arm activities in patients with COPD
}

\author{
Kenneth Meijer ${ }^{1}$, Janneke Annegarn' ${ }^{1}$, Valéria Lima Passos², Hans H. Savelberg ${ }^{1}$, \\ Annemie M. Schols ${ }^{3}$, Emiel F. Wouters ${ }^{3,4}$ and Martijn A. Spruit ${ }^{4}$
}

\begin{abstract}
Affiliations:
'Dept of Human Movement Science, NUTRIM School for Nutrition, Toxicology and Metabolism, Maastricht University Medical Centre, Maastricht, The Netherlands.

${ }^{2}$ Dept of Methodology and Statistics, Maastricht University, Maastricht, The Netherlands.

${ }^{3}$ Dept of Respiratory Medicine, NUTRIM School for Nutrition, Toxicology and Metabolism, Maastricht University Medical Centre, Maastricht, The Netherlands.

${ }^{4}$ Dept of Research and Education, ClRO+, Centre of Expertise for Chronic Organ Failure, Horn, The Netherlands.

\section{Correspondence:}

K. Meijer, Dept of Human Movement Science, NUTRIM School for Nutrition, Toxicology and Metabolism, Maastricht University Medical Centre, P0 Box 616, 6200 Maastricht, the Netherlands.

E-mail: kenneth.meijerdmaastrichtuniversity.nl
\end{abstract}

ABSTRACT Arm activities are required for maintenance of self-care and independent living. This study aimed to investigate whether and to what extent arm activities of daily living (ADL) in chronic obstructive pulmonary disease (COPD) patients differ compared to healthy controls and the extent to which they perform arm ADL at a relatively higher upper limb muscle effort.

Daily arm and leg activities were assessed using accelerometers in the home environment (COPD: $\mathrm{n}=21$, healthy: $n=24$; part 1 ). The relative efforts of the trapezius, deltoid and biceps muscles were studied using electromyography during domestic arm ADL in a laboratory setting (COPD: $n=17$, healthy: $n=15$; part 2).

After correction for walking time, the time spent on arm ADL was similar between COPD patients and healthy control subjects $(\mathrm{p}=0.52)$, while the intensity of arm activities was lower in COPD patients $(\mathrm{p}=0.041)$. In the laboratory setting, arm ADL were performed at a lower intensity by COPD patients, while the trapezius muscle effort was significantly higher during several arm ADL compared to healthy control subjects $(\mathrm{p}<0.05)$.

COPD patients have a similar duration of arm ADL compared to healthy subjects after correction for walking time, but perform arm activities at a lower intensity. Moreover, patients perform some arm ADL at a relatively higher muscle effort.

@ERSpublications

COPD patients perform daily arm activities less intensively than healthy subjects but require more muscle effort http://ow.ly/rSv25

For editorial comments see page 1551.

This article has supplementary material available from www.erj.ersjournals.com

Received: May 132013 | Accepted after revision: Dec 112013 | First published online: Feb 202014

Conflict of interest: None declared.

Copyright (OERS 2014 


\section{Introduction}

Physical inactivity is a major risk factor for accelerated disability [1], and a worse prognosis in patients with chronic obstructive pulmonary disease (COPD) [2, 3]. Regular physical activities, such as walking, are problematic in patients with COPD [4,5]. Moreover, simple domestic activities of daily living (ADL) that involve the arms, such as cleaning and dressing, can also be impaired and symptom inducing $[5,6]$. These impairments are of concern because they limit a patients' independence during everyday life [7, 8].

To date, characteristics of arm ADL in COPD are not well-described in the peer-reviewed literature. Patients with COPD experience a relatively high metabolic load and dyspnoea during the performance of self-paced domestic arm ADL compared to healthy control subjects [6]. Moreover, arm elevation in patients with COPD has been associated with loss of vital capacity [9-16]. Therefore, arm activities, especially when involving arm elevation, are demanding for accessory inspiratory muscles, such as the trapezius muscle [17]. Consequently, patients with COPD may reduce arm ADL to reduce the burden of task-related dyspnoea. Conversely, a preservation of deltoid and biceps muscle endurance has been reported in patients with COPD, which can possibly be explained by the maintenance of arm ADL [18-21].

Assessment of arm activities in the home environment of COPD patients is essential to better understand the problems associated with ADL. This requires a validated arm accelerometer and detailed electromyographic evaluation of muscle function during daily arm activities (unpublished observations). Therefore, the present study aimed to quantify daily arm activities in patients with COPD and healthy control subjects. Moreover, effort of the trapezius, biceps and deltoid muscles were assessed during the performance of daily arm activities in a laboratory setting to explore the mechanisms underlying the decreased arm activity in COPD. It was hypothesised that daily arm activities were preserved as they are still required for maintenance of self-care and independent living and that this comes at the expense of a larger muscle effort.

\section{Methods}

\section{Study description}

This study consisted of two parts. Part 1 was performed to assess arm and leg activity in daily life in participants' home environment. Part 2 was conducted to study relative effort of the trapezius, deltoid and biceps muscles during daily arm activities in a laboratory setting. In both parts, arm and leg activities were measured by two accelerometers (worn on the upper arm just above the elbow, and on the upper leg just above the knee). Leg activities (e.g. walking) result in whole-body movement, including the arms. To compare activities of the arms only (with no leg movements involved) between COPD and healthy subjects (part 1 of the study), arm activities were not counted when leg activity was measured simultaneously.

\section{Patient selection}

All patients were recruited at CIRO+, the Center of Expertise for Chronic Organ Failure in Horn, the Netherlands [22]. Inclusion criteria were: 1) diagnosis of COPD according to criteria determined by the Global Initiative for Chronic Obstructive Lung Disease; 2) no exacerbation in the past 4 weeks; and 3) absence of pathological conditions that could impair physical activities in daily life, such as stroke. Healthy subjects were relatives of employees and students of CIRO+ or healthy spouses of patients at CIRO+. Inclusion criteria were the same as the COPD group, with the addition of normal spirometry results and no use of physician-prescribed drugs. Five patients with COPD and 15 healthy subjects participated in both parts of the study. Not all subjects participated in both parts of the study because they were separate studies performed parallel to one another. The study was approved by the medical ethical committee of Maastricht University Medical Centre, Maastricht, the Netherlands (part 1: MEC 10-3-086; part 2: MEC 10-3-077). All participants gave written informed consent prior to inclusion.

\section{Instrumentation}

Two triaxial accelerometers (CIRO Activity Monitor (CAM); Maastricht Instruments B.V., Maastricht) were used in both parts of the study to accurately assess activities and postures. The CAM was used as it can assess both leg activity (CAMleg) [23] and arm activity (CAMarm) (unpublished observation). In brief, CAMleg measures the time spent walking (or engaged in other dynamic activities), in weight bearing postures (standing) and non-weight bearing postures (sitting and lying), as well as movement intensity during walking. CAMarm measures the intensity and elevation of the arm at each second, which will then be categorised into three levels of movement intensity (posture: holding still; medium: moving slow; high: moving fast) and arm elevation (low: $\leqslant 45^{\circ}$; medium: $45-90^{\circ}$; high: $>90^{\circ}$ ) (fig. 1). Matlab software and algorithms (MathWorks, Natick, MA, USA) were used to calculate the signal magnitude area as a measure of arm intensity and the mean low pass signal in the longitudinal direction as a measure of arm elevation [24]. A hierarchical classification scheme was used to differentiate between three levels of arm elevation and arm intensity. 

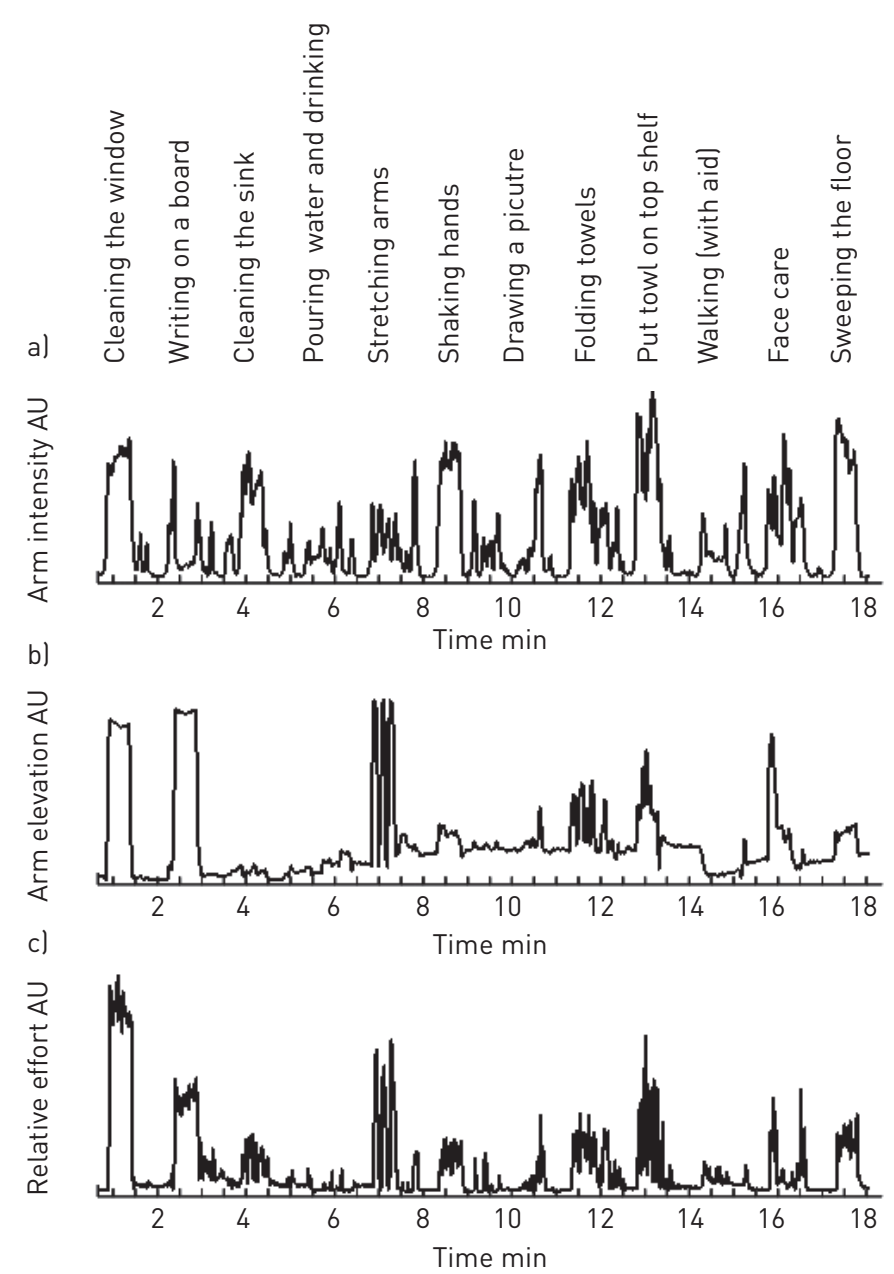

FIGURE 1 Example of a) arm intensity, b) arm elevation and c) relative effort of the deltoid muscle obtained during the protocol in a controlled setting. Data were obtained from a random chronic obstructive pulmonary disease patient. The patient was sitting during rest after the activities "drinking water", "stretching arms", "shaking hands", "drawing a picture", "folding towels" and "face care". During rest the patient was leaning on the table or walking aid and showed an increased arm elevation. The first activity (cleaning the window) is a combination of high intensity and high elevation of the arm, resulting in the highest relative muscle effort within this patient. The second activity (writing on a board) shows the effect of high elevation in combination with a low intensity of the arm on relative muscle effort. The third activity (cleaning the sink) shows the effect of low elevation in combination with a high intensity of the arm on relative muscle effort. AU: arbitrary units.

The following were assessed: pulmonary function (forced expiratory volume in $1 \mathrm{~s}$ (FEV1), forced vital capacity (FVC), and FEV1/FVC [25]); the degree of dyspnoea and fatigue [6]; muscle strength and endurance of the quadriceps muscle and biceps muscle using a dynamometer [26] (part 1 only); and maximal and task-related effort of the trapezius, biceps and deltoid muscle using a Programmable Ambulant Signal AcQuisition (PASAQ; Maastricht Instruments B.V.) system (part 2 only) [27]. The methods and equipment are described in more detail in the online supplement and figures E1-E6.

\section{Assessment}

\section{Part 1: in daily life}

Participants were asked to wear the CAMarm and CAMleg simultaneously for six full days during waking hours. The CAM is not waterproof, therefore subjects had to remove it while showering or swimming. Both sensors were programmed to automatically record from $07.00 \mathrm{~h}$ to $19.00 \mathrm{~h}$. The evening hours (after $19.00 \mathrm{~h}$ ) were not included in the analysis because it is probable that, in general, elderly people perform considerably fewer activities during this period compared with the other periods of the day [28]. The mean time for which the sensors were worn was similar between patients with COPD and healthy control subjects $(5.3 \pm 0.8$ days for $9.9 \pm 1.2 \mathrm{~h}$ and $5.6 \pm 0.7$ days for $10.4 \pm 0.9 \mathrm{~h}$, respectively), and there were no 
differences in wearing time between the arm and leg. Since the subjects were not all measured for exactly the same duration (due to the fact that some patients woke up after $07.00 \mathrm{~h}$ ), the time spent in different activity categories were normalised for $10 \mathrm{~h}$ per day and averaged over the number of days worn.

\section{Part 2: in the laboratory setting}

Participants performed 12 domestic activities of daily life at the Dept of Occupational Therapy, CIRO+ (fig. 1). The activities were performed in the same order by all participants and they were asked to carry out activities as they would do during daily life, i.e. at their preferred intensity and level of elevation of the arm. Each activity lasted for $30 \mathrm{~s}$ and was followed by 30-60 s of rest. Time was monitored using a stopwatch. $5 \mathrm{~s}$ at the beginning and end of each activity were excluded from further analyses. During the protocol, arm elevation, arm intensity and effort of the biceps, deltoid and trapezius muscles were assessed using electromyograph (EMG) and expressed as percentage of maximal muscle effort.

Relative muscle effort was measured using surface EMG and a PASAQ. A common ground electrode was placed on the ulnar styloid process. The cables from the electrodes were taped to the skin and placed into the PASAQ, which the participant wore in a small backpack (fig. E5). The signal from the electrodes was sampled at $1000 \mathrm{~Hz}$. Matlab software and algorithms were used to calculate the muscle effort as described previously [27]. For this purpose, signals were rectified, smoothed at a time constant of $0.01 \mathrm{~s}$ and downsampled by a factor of 100 . A value representing muscle effort was obtained for each second by averaging over 10 samples. To obtain the relative muscle effort these values were normalised for each subject in such a way that they expressed the percentage of the participant's maximum voluntary effort assessed after the protocol (fig. E3).

To assess maximal muscle effort, participants were asked for a maximal voluntary effort for $3 \mathrm{~s}$ for each muscle twice, with $30 \mathrm{~s}$ rest in between. For the trapezius muscle, participants were asked sit straight, grasp the chair seat and pull as hard as possible toward the ceiling (fig. E6, position 1). For the biceps muscle, participants were asked to sit in front of a table fixed to the wall, and push with the wrist against the table as hard as possible toward the ceiling (fig. E6, position 2). For the deltoid muscle, participants were asked to sit with their elbow against the table and push as hard as possible toward the wall (fig. E6, position 3).

\section{Statistics}

Continuous and categorical variables are presented as mean $\pm \mathrm{SD}$ or proportion, respectively. Relative muscle effort of all three muscles displayed skewedness to the right in their frequency distributions, and were therefore $\log _{10}$ transformed. Data from COPD patients and healthy control subjects were compared using the independent t-test or Chi-squared test, as appropriate. Arm movement intensity can be influenced by leg activity. Therefore, data were re-analysed after excluding walking time.

In part 2 of this study, variables (e.g. muscle effort, intensity and elevation of the arm) for each activity in the protocol were first averaged over the repeated measures (20 samples per activity per subject) and then compared between groups using an independent t-test. In addition, relative muscle effort, intensity and elevation levels of the arm were compared between groups. To account for the nested structure of the data (repeated measures within subjects over different controlled conditions), a random intercept model analysis was conducted (mixed model). Subjects were the random factor, and sex, group (COPD versus healthy), arm intensity (holding still versus moving slow versus moving fast) and arm elevation (low versus medium versus high) were the fixed effects. A priori, the level of significance was set at $\mathrm{p}<0.05$. Data analysis was performed using SPSS (version 15.0; Chicago, IL, USA).

\section{Results}

Part 1: in daily life

Subjects' characteristics

Daily arm and leg activities were assessed in 21 patients with COPD and 24 healthy control subjects. Patients were suffering from mild-to-very severe COPD [29]. Sex distribution, age, weight and height were similar between groups. COPD patients had lower quadriceps muscle strength and endurance, while no significant differences were found for the biceps muscle (table 1). The degree of fatigue and dyspnoea are described in figure E7.

Daily leg activity and postures

Patients with COPD spent more time sitting and/or lying (COPD: $394 \pm 66 \mathrm{~min}$; healthy: $328 \pm 77 \mathrm{~min}$; $\mathrm{p}<0.01$ ) and less time walking (COPD: $86 \pm 39 \mathrm{~min}$; healthy: $134 \pm 53 \mathrm{~min} ; \mathrm{p}<0.01$ ). Time spent standing was similar between groups (COPD: $120 \pm 38 \mathrm{~min}$; healthy: $136 \pm 49 \mathrm{~min} ; \mathrm{p}=0.11$ ). Moreover, intensity of walking was lower in patients with COPD (COPD: $38.2 \pm 3.7$ arbitrary units (AU); healthy: $47.3 \pm 7.7$ AU; $\mathrm{p}<0.01)$. 
TABLE 1 Patient characteristics for part 1: activities in daily life

\begin{tabular}{|c|c|c|c|c|}
\hline & COPD & Healthy & $95 \% \mathrm{Cl}$ & p-value \\
\hline Subjects $\mathrm{n}$ & 21 & 24 & & \\
\hline Males \% & 76.2 & 70.8 & $-0.3-0.2$ & 0.746 \\
\hline Age years & $64.1 \pm 7.7$ & $62.4 \pm 6.2$ & $-2.5-5.9$ & 0.410 \\
\hline Weight kg & $79.2 \pm 14.5$ & $77.3 \pm 12.8$ & $-6.2-10.2$ & 0.631 \\
\hline Height $\mathrm{m}$ & $1.73 \pm 0.08$ & $1.74 \pm 0.09$ & $-0.06-0.04$ & 0.682 \\
\hline $\begin{array}{l}\text { BMI } \mathrm{kg} \cdot \mathrm{m}^{-2} \\
\text { GOLD stage } 1 / 2 / 3 / 4 \mathrm{n}\end{array}$ & $\begin{array}{c}26.5 \pm 4.9 \\
4 / 10 / 5 / 2\end{array}$ & $25.4 \pm 3.3$ & $-1.4-3.6$ & 0.379 \\
\hline FEV 1 L & $1.73 \pm 0.66$ & $3.53 \pm 0.54$ & $-2.2--1.4$ & $<0.001$ \\
\hline FEV $1 \%$ predicted & $57.8 \pm 18.9$ & $117.6 \pm 19.2$ & $-71.3--48.4$ & $<0.001$ \\
\hline FVC L & $3.76 \pm 0.92$ & $4.42 \pm 0.50$ & $-1.11--0.19$ & 0.007 \\
\hline FVC $\%$ predicted & $99.6 \pm 17.3$ & $118.6 \pm 23.4$ & $-31.6--6.6$ & 0.004 \\
\hline Tiffeneau index \% & $0.46 \pm 0.14$ & $0.80 \pm 0.06$ & $-0.41--0.28$ & $<0.001$ \\
\hline \multicolumn{5}{|c|}{ Quadriceps femoris muscle } \\
\hline Strength $\mathrm{Nm}$ & $104 \pm 37$ & $134 \pm 39$ & $-52--7$ & 0.012 \\
\hline Endurance s & $38 \pm 14$ & $49 \pm 12$ & $-19--4$ & 0.005 \\
\hline \multicolumn{5}{|l|}{ Biceps brachii muscle } \\
\hline Strength $\mathrm{Nm}$ & $49 \pm 15$ & $57 \pm 15$ & $-18-0$ & 0.056 \\
\hline Endurance s & $60 \pm 46$ & $76 \pm 28$ & $-38-7$ & 0.170 \\
\hline
\end{tabular}

Data are presented as mean \pm SD, unless otherwise stated. COPD: chronic obstructive pulmonary disease; BMI: body mass index; GOLD: Global Initiative for Chronic Obstructive Lung Disease; FEV1: forced expiratory volume in $1 \mathrm{~s}$; FVC: forced vital capacity.

\section{Daily arm activity and postures}

Patients with COPD spent more time on arm postures (COPD: $451 \pm 57$ min; healthy: $400 \pm 62 \mathrm{~min}$; $\mathrm{p}<001)$ and less time on arm activities (COPD $149 \pm 57$ min; healthy: $200 \pm 62 \mathrm{~min} ; \mathrm{p}<0.01)$ compared to healthy control subjects. Differences in arm activity time were only present during low arm elevation, which is the typical arm category during walking. After correction for walking time, the time spent on daily arm activities was similar between patients with COPD and healthy control subjects. Nevertheless, the intensity of arm activities was lower in patients with COPD (COPD: $30.5 \pm 2.5$ AU; healthy $33.6 \pm 3.6 \mathrm{AU} ; \mathrm{p}=0.002$ ), also after correction of walking time $(\mathrm{p}=0.041)$ (table 2$)$.

\section{Part 2: in the laboratory setting}

Subjects' characteristics

Relative muscle effort during domestic daily arm activities was studied in 17 patients with COPD and 15 healthy control subjects. Sex distribution, age, weight and height were comparable between groups (table 3). A representative example of analysed EMG and acceleration signals obtained from a patient with COPD

TABLE 2 Characteristics of daily arm activities

\begin{tabular}{lcccc} 
& COPD & Healthy & $\mathbf{9 5 \%}$ Cl & p-value \\
\hline After correction for walking time & & & & \\
$\quad$ Arm postures min & $436.1 \pm 56.6$ & $382.3 \pm 65.5$ & $16.8-90.9$ & 0.005 \\
$\quad$ Arm activity min & $77.2 \pm 28.2$ & $82.6 \pm 28.4$ & $-22.5-11.6$ & 0.524 \\
Arm activity per category min" & & & & \\
$\quad$ High intensity, high elevation & $0.4 \pm 0.4$ & $0.4 \pm 0.3$ & $-0.2-0.2$ & 0.719 \\
$\quad$ High intensity, medium elevation & $4.7 \pm 2.1$ & $5.5 \pm 3.0$ & $-2.4-0.8$ & 0.302 \\
$\quad$ High intensity, low elevation & $3.4 \pm 2.2$ & $5.0 \pm 3.6$ & $-3.4-0.2$ & 0.072 \\
$\quad$ Medium intensity, high elevation & $1.9 \pm 1.5$ & $1.3 \pm 0.5$ & $-0.1-1.3$ & 0.095 \\
$\quad$ Medium intensity, medium elevation & $22.4 \pm 9.7$ & $22.9 \pm 8.3$ & $-5.9-4.9$ & 0.858 \\
$\quad$ Medium intensity, low elevation & $44.4 \pm 17.5$ & $47.5 \pm 17.2$ & $-13.6-7.3$ & 0.546 \\
Activity intensity AU & $28.5 \pm 1.7$ & $29.8 \pm 2.4$ & $-2.6--0.1$ & 0.041 \\
\hline
\end{tabular}

Data are presented as mean $\pm S D$, unless otherwise stated. COPD: chronic obstructive pulmonary disease; $A U$ : arbitrary units. \#: low intensity is considered as posture and therefore not included in the table as a subcategory of arm activities. 
during the protocol is presented in figure 1. In a few cases, EMG data of the trapezius muscle (COPD n=2) and biceps muscle (COPD: $n=3$; healthy: $n=1$ ) were excluded due to loss of electrode contact and/or movement artefacts. The degree of fatigue and dyspnoea are described in figure E9.

Activities and relative muscle effort during arm ADL protocol

Patients with COPD performed seven of the 12 activities of the protocol at a lower movement intensity compared to healthy control subjects, while the degree of arm elevation was similar between groups (table 4). Six activities of the trapezius muscle (writing on a board, shaking hands, folding towels, put towels on the shelf, walking at preferred speed and sweeping the floor) and one activity of the biceps muscle (folding towels) were performed at a higher proportion of the maximum muscle effort compared to healthy control subjects. No differences were observed for the deltoid muscle (table 5).

\section{Activity categorisation during arm ADL protocol}

Activities were categorised to compare relative muscle effort between groups, intensity levels and elevation levels of the arm (fig. 2, tables E1 and E2). Time spent in the high intensity categories was lower for COPD patients (table E5). Significant three-way interactions between group, elevation and intensity were detected, indicating that differences between patients with COPD and healthy control subjects depended on the levels of both intensity and elevation of the arm. Lower intensity and lower arm elevation was accompanied with lower relative effort for all muscles in both groups. The group effects (COPD versus healthy) were not the same for all muscles. For each level of orientation and intensity, COPD patients clearly showed relatively higher effort of the trapezius muscle, while no consistent differences could be found for the biceps and deltoid muscle.

Within the typical arm category for walking (medium intensity in combination with a low arm elevation), arm muscle efforts (of all muscles) were significantly lower with the presence of leg activity (leg muscles initiated the movement and not the arm muscle) compared to without the presence of leg activity $(\mathrm{p}<0.05$ (fig. E10).

\section{Discussion}

This study is the first to characterise daily arm activities and associated relative effort of upper body muscles in patients with mild-to-severe COPD. Patients with COPD spent more time in arm postures versus arm activities. Furthermore, the arm movement intensity was lower compared to healthy control subjects. After correction for walking, the time spent on daily arm activities was similar between patients with COPD and healthy control subjects, while the intensity of arm movements remained significantly lower in patients with COPD. Also, in a laboratory setting the majority of the arm ADL were performed with lower movement intensity by the patients with COPD. Despite this, patients with COPD had a higher task-related trapezius muscle effort during several domestic ADL compared to healthy control subjects.

Many COPD patients entering pulmonary rehabilitation experience problems with arm activities [5]. This study shows that in daily life patients with COPD spent the same amount of time on arm activities during sitting and standing as healthy peers, although arm activities are poorly tolerated by patients with COPD

\section{TABLE 3 Patient characteristics for part 2: activities in the laboratory}

\section{COPD}

Subjects $\mathbf{n}$
Males \%
Age years
Weight $\mathbf{k g}$
Height $\mathrm{m}$
BMI $\mathrm{kg} \cdot \mathrm{m}^{-2}$
GOLD stage $1 / 2 / 3 / 4 \mathrm{n}$
FEV $1 \mathrm{~L}$
FEV $1 \%$ predicted
FVC L
FVC \% predicted
Tiffeneau index $\%$

18
55.6
$62.4 \pm 8.1$
$73.7 \pm 12.9$
$1.70 \pm 0.09$
$25.5 \pm 4.5$
$3 / 5 / 8 / 2$
$1.41 \pm 0.65$
$50.1 \pm 20.1$
$3.40 \pm 0.90$
$96.0 \pm 16.3$
$0.42 \pm 0.15$

Data are presented as mean \pm SD, unless otherwise stated. COPD: chronic obstructive pulmonary disease; BMI: body mass index; GOLD: Global Initiative for Chronic Obstructive Lung Disease; FEV1: forced expiratory volume

in $1 \mathrm{~s}$; FVC: forced vital capacity.

$\begin{array}{ccc} & & \\ 15 & & \\ 53.3 & -0.4-0.3 & 0.588 \\ 65 \pm 7 & -7.8-2.9 & 0.351 \\ 74.5 \pm 12.0 & -8.7-9.1 & 0.968 \\ 1.74 \pm 0.09 & -0.11-0.02 & 0.213 \\ 24.1 \pm 2.9 & -1.37-4.14 & 0.313 \\ & & \\ 3.48 \pm 0.58 & -2.51--1.63 & <0.001 \\ 118.9 \pm 16.0 & -81.8--55.7 & <0.001 \\ 4.39 \pm 0.60 & -1.55--0.44 & 0.001 \\ 124.7 \pm 24.8 & -43.4--14.0 & <0.001 \\ 0.79 \pm 0.05 & -0.45--0.29 & <0.001\end{array}$


TABLE 4 Mean and difference between chronic obstructive pulmonary disease (COPD) and healthy patients in arm intensity and elevation, and leg intensity for each activity

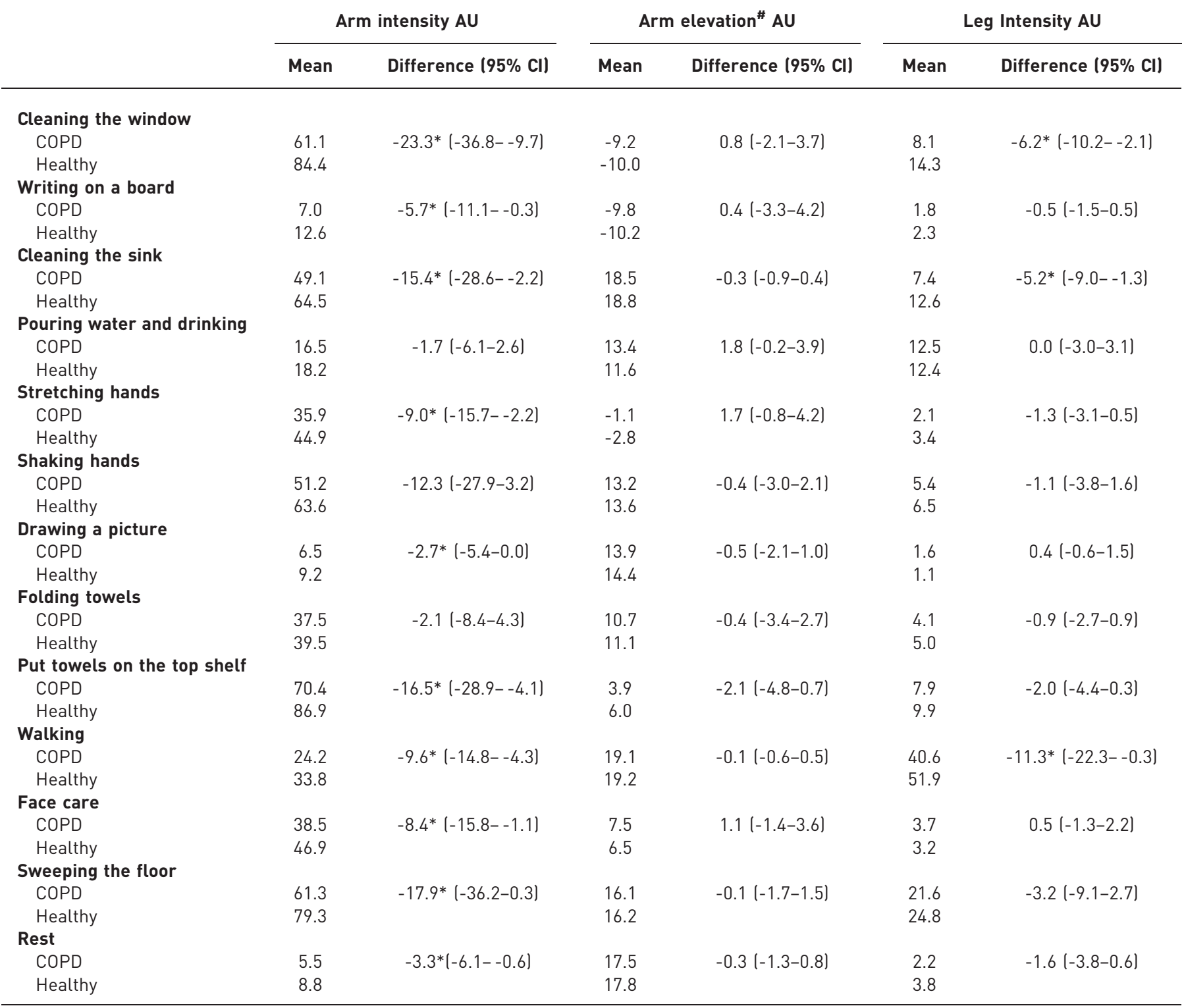

AU: arbitrary units. " : calculated using the mean low pass signal in the longitudinal direction, lower longitudinal direction means higher arm elevation. ${ }^{*}: \mathrm{p}<0.05$ between COPD and healthy patients.

[9]. Arm activity time may be preserved because many arm activities are necessary for independent living (i.e. preparing food and self care) $[7,8]$.

Although patients with COPD spent as much time on arm activities during sitting and standing as healthy control subjects, other characteristics of daily arm activities differed compared to the control group. Indeed, arm ADL were performed at a significantly lower intensity. Possibly, the lower intensity indicates that patients with COPD perform less daily tasks involving the arms or they perform them at a lower frequency [30].

Measurements in a laboratory setting allowed for a more detailed analysis regarding performance of daily activities in relation to maximal muscle effort. Similar to the daily life situation, patients with COPD generally performed the instructed activities with lower arm movement intensity. However, the relative effort compared to maximum of the shoulder/arm muscles was similar or higher compared to healthy control subjects (tables E1 and E2). Increased relative muscle effort during specific ADL was found in the trapezius muscle of patients with COPD, while relative muscle effort for the biceps and deltoid muscles were 


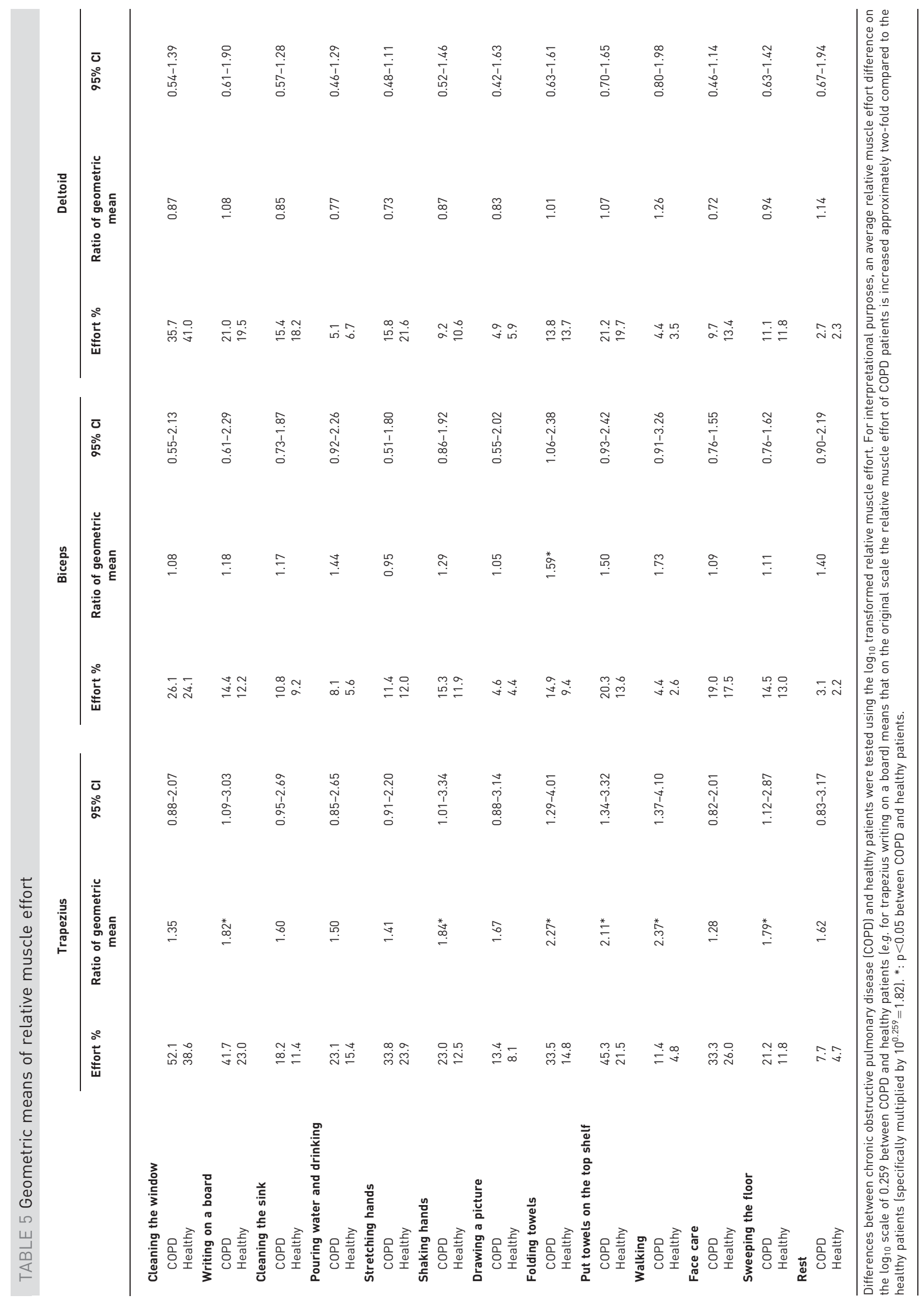



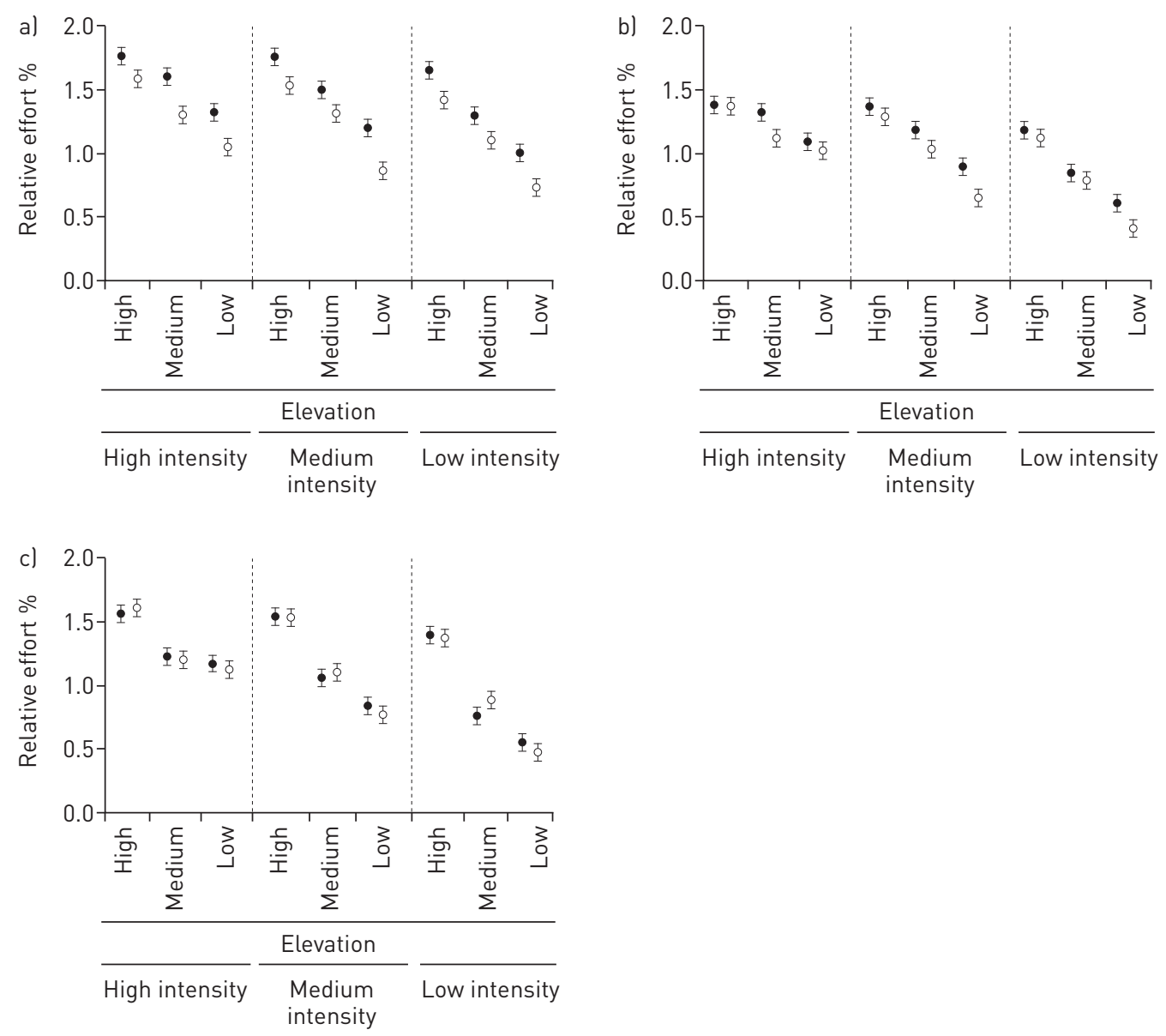

FIGURE 2 Log transformed relative muscle effort of the a) trapezius, b) biceps brachii and c) deltoid muscles at different levels of arm elevation and arm intensity for chronic obstructive pulmonary disease (COPD) patients (closed circles) and healthy patients (open circles). An average relative muscle effort difference on the $\log _{10}$ scale of 0.332 between COPD and healthy patients (e.g. for trapezius low elevation/medium intensity) means that on the original scale the relative muscle effort of COPD patients is increased approximately two-fold compared to the healthy patients (specifically multiplied by $\left.10^{0.332}=2.147\right)$ (table E4). Error bars represent SEM.

similar to those of healthy control subjects. These results suggest that COPD patients perform daily arm activities at a similar relative biceps and deltoid muscle effort as healthy control subjects by lowering the intensity of their arm movements. Moreover, the relatively high effort of the trapezius muscle is probably explained by its inspiratory function [17]. Indeed, it is in line with the finding that during walking, which increases ventilator requirements [31], relative effort of the trapezius muscle of patients with COPD was much higher compared to healthy control subjects. Furthermore, differences in trapezius muscle effort between groups increased with arm elevation. This can be explained by previous findings showing that arm elevation in COPD patients is associated with changes in lung volumes and loss of vital capacity [9-16].

Some methodological considerations need to be made. This study was performed in a group of patients with COPD entering pulmonary rehabilitation. Nevertheless, time spent in leg weight-bearing postures (e.g. sitting and lying) are similar to values reported by PITTA et al. [28] (66\% versus 64\%, respectively). None of the patients with COPD used walking aids, such as a rollator, in daily life, which will most probably affect assessment of arm ADL [32]. Participants did not wear the device for exactly the same time-period for various reasons (e.g. woke up late, swimming, etc.). Nevertheless, all participants were measured for at least 3 days, which is considered to be sufficient for a valid assessment of activity patterns [28].

The degree of airflow limitation was rather broad (FEV1 ranged 20-88\% predicted and 21-81\% pred for parts 1 and 2, respectively). The mean FEV1, however, was $>50 \%$ pred in both study samples. Whether and to what extent the differences between patients with COPD and healthy control subjects would be larger in patients with only very severe COPD remains unknown. Arm exercise programmes can effectively increase arm exercise capacity $[33,34]$. Moreover, a reduction of the task-related burden needs to be considered by learning and applying energy conservation techniques [35]. These techniques can result in significant 
decreases in task-related oxygen uptake during the performance of domestic ADL in patients with COPD [30]. Future studies should evaluate the effects of different tailored interventions on arm ADLs in patients with COPD. However, it seems reasonable to hypothesise that the abovementioned interventions may improve arm muscle capacity and reduce the burden of ADL and, in turn, reduce task-related dyspnoea. For example, 15 sessions of unsupported upper extremity exercise training improved ADL and reduced the perception of fatigue in patients with COPD up to 6 months after the intervention [36]. Whether and to what extent pulmonary rehabilitation programmes, including unsupported arm exercises and energy conservation techniques, may also improve daily arm activities in terms of duration per day, arm elevation level and/or movement intensity and, in turn, patients' autonomy remains currently unknown.

In conclusion, patients with COPD have similar duration of daily arm activities compared to healthy control subjects after correction for walking, but perform arm activities at a lower intensity. Moreover, patients perform some daily arm activities at a higher proportion of their maximum trapezius muscle effort. Future studies are warranted to assess the effects of a tailored pulmonary rehabilitation programme on arm $\mathrm{ADL}$ in patients with COPD.

\section{Acknowledgements}

The authors are grateful to the volunteers who participated in the present study. Moreover Y. van Dooren, N. Ritsema and P. Willems (Dept of Human Movement Science, NUTRIM School for Nutrition, Toxicology and Metabolism, Maastricht University Medical Centre, Masstricht, the Netherlands) are gratefully acknowledged for their help with data collection and electronic data processing.

\section{References}

$1 \quad$ Katz $\mathrm{P}$, Chen $\mathrm{H}$, Omachi TA, et al. The role of physical inactivity in increasing disability among older adults with obstructive airway disease. J Cardiopulm Rehabil Prev 2011; 31: 193-197.

2 Waschki B, Kirsten A, Holz O, et al. Physical activity is the strongest predictor of all-cause mortality in patients with COPD: a prospective cohort study. Chest 2011; 140: 331-342.

3 Garcia-Aymerich J, Lange P, Benet M, et al. Regular physical activity reduces hospital admission and mortality in chronic obstructive pulmonary disease: a population based cohort study. Thorax 2006; 61: 772-778.

4 Annegarn J, Spruit MA, Savelberg HH, et al. Differences in walking pattern during 6-min walk test between patients with COPD and healthy subjects. PLoS One 2012; 7: e37329.

5 Annegarn J, Meijer K, Passos VL, et al. Problematic activities of daily life are weakly associated with clinical characteristics in COPD. J Am Med Dir Assoc 2012; 13: 284-290.

6 Vaes AW, Wouters EF, Franssen FM, et al. Task-related oxygen uptake during domestic activities of daily life in patients with COPD and healthy elderly subjects. Chest 2011; 140: 970-979.

7 Guccione AA, Felson DT, Anderson JJ, et al. The effects of specific medical conditions on the functional limitations of elders in the Framingham Study. Am J Public Health 1994; 84: 351-358.

8 Rodriguez Gonzalez-Moro JM, de Lucas Ramos P, Izquierdo Alonso JL, et al. Impact of COPD severity on physical disability and daily living activities: EDIP-EPOC I and EDIP-EPOC II studies. Int J Clin Pract 2009; 63: 742-750.

9 Dolmage TE, Maestro L, Avendano MA, et al. The ventilatory response to arm elevation of patients with chronic obstructive pulmonary disease. Chest 1993; 104: 1097-1100.

10 Celli BR, Rassulo J, Make BJ. Dyssynchronous breathing during arm but not leg exercise in patients with chronic airflow obstruction. N Engl J Med 1986; 314: 1485-1490.

11 Lebzelter J, Klainman E, Yarmolovsky A, et al. Relationship between pulmonary function and unsupported arm exercise in patients with COPD. Monaldi Arch Chest Dis 2001; 56: 309-314.

12 Martinez FJ, Couser JI, Celli BR. Respiratory response to arm elevation in patients with chronic airflow obstruction. Am Rev Respir Dis 1991; 143: 476-480.

13 Baarends EM, Schols AM, Slebos DJ, et al. Metabolic and ventilatory response pattern to arm elevation in patients with COPD and healthy age-matched subjects. Eur Respir J 1995; 8: 1345-1351.

14 Criner GJ, Celli BR. Effect of unsupported arm exercise on ventilatory muscle recruitment in patients with severe chronic airflow obstruction. Am Rev Respir Dis 1988; 138: 856-861.

15 Gigliotti F, Coli C, Bianchi R, et al. Arm exercise and hyperinflation in patients with COPD: effect of arm training. Chest 2005; 128: 1225-1232.

16 Epstein SK, Celli BR, Williams J, et al. Ventilatory response to arm elevation. Its determinants and use in patients with chronic obstructive pulmonary disease. Am J Respir Crit Care Med 1995; 152: 211-216.

17 American Thoracic Society/European Respiratory Society. ATS/ERS statement on respiratory muscle testing. Am J Respir Crit Care Med 2002; 166: 518-624.

18 Franssen FM, Broekhuizen R, Janssen PP, et al. Limb muscle dysfunction in COPD: effects of muscle wasting and exercise training. Med Sci Sports Exerc 2005; 37: 2-9.

19 Franssen FM, Wouters EF, Baarends EM, et al. Arm mechanical efficiency and arm exercise capacity are relatively preserved in chronic obstructive pulmonary disease. Med Sci Sports Exerc 2002; 34: 1570-1576.

20 Clark CJ, Cochrane LM, Mackay E, et al. Skeletal muscle strength and endurance in patients with mild COPD and the effects of weight training. Eur Respir J 2000; 15: 92-97.

21 Gea JG, Pasto M, Carmona MA, et al. Metabolic characteristics of the deltoid muscle in patients with chronic obstructive pulmonary disease. Eur Respir J 2001; 17: 939-945.

22 Spruit MA, Vanderhoven-Augustin I, Janssen PP, et al. Integration of pulmonary rehabilitation in COPD. Lancet 2008; 371: 12-13.

23 Annegarn J, Spruit MA, Uszko-Lencer NH, et al. Objective physical activity assessment in patients with chronic organ failure: a validation study of a new single-unit activity monitor. Arch Phys Med Rehabil 2011; 92: 1852-1857. 

classification techniques. Physiol Meas 2009; 30: R1-33.

25 Quanjer PH, Tammeling GJ, Cotes JE, et al. Lung volumes and forced ventilatory flows. Eur Respir J 1993; 6: Suppl. $16,5-40$.

26 Allaire J, Maltais F, Doyon JF, et al. Peripheral muscle endurance and the oxidative profile of the quadriceps in patients with COPD. Thorax 2004; 59: 673-678.

27 Theou O, Jones GR, Vandervoort AA, et al. Daily muscle activity and quiescence in non-frail, pre-frail, and frail older women. Exp Gerontol 2010; 45: 909-917.

28 Pitta F, Troosters T, Spruit MA, et al. Characteristics of physical activities in daily life in chronic obstructive pulmonary disease. Am J Respir Crit Care Med 2005; 171: 972-977.

29 Rabe KF, Hurd S, Anzueto A, et al. Global strategy for the diagnosis, management, and prevention of chronic obstructive pulmonary disease: GOLD executive summary. Am J Respir Crit Care Med 2007; 176: $532-555$.

30 Velloso M, Jardim JR. Study of energy expenditure during activities of daily living using and not using body position recommended by energy conservation techniques in patients with COPD. Chest 2006; 130: $126-132$.

31 Marquis N, Debigaré R, Bouyer L, et al. Physiology of walking in patients with moderate to severe chronic obstructive pulmonary disease. Med Sci Sports Exerc 2009; 41: 1540-1548.

32 Vaes AW, Annegarn J, Meijer K, et al. The effects of a "new" walking aid on exercise performance in patients with COPD: a randomized crossover trial. Chest 2012; 141: 1224-1232.

33 Janaudis-Ferreira T, Hill K, Goldstein RS, et al. Resistance arm training in patients with COPD: a randomized controlled trial. Chest 2011; 139: 151-158.

34 McKeough ZJ, Bye PT, Alison JA. Arm exercise training in chronic obstructive pulmonary disease: a randomised controlled trial. Chron Respir Dis 2012; 9: 153-162.

35 Velloso M, Jardim JR. Functionality of patients with chronic obstructive pulmonary disease: energy conservation techniques. J Bras Pneumol 2006; 32: 580-586.

36 Costi S, Crisafulli E, Antoni FD, et al. Effects of unsupported upper extremity exercise training in patients with COPD: a randomized clinical trial. Chest 2009; 136: 387-395. 